\title{
Die spätglaziale Vegetationsgeschichte im oberen Illergebiet [Allgäu/Bayern]
}

\author{
Philipp Stojakowits, Arne Friedmann, Andrea Bull
}

How to cite:

Stojakowits, Ph., Friedmann, A., Bull, A. (2014): Die spätglaziale Vegetationsgeschichte im oberen Illergebiet (Allgäu/Bayern). E\&G Quaternary Science Journal, 63 (2): 130-142. DOI: 10.3285/eg.63.2.02

Kurzfassung:

\begin{abstract}
Anhand dreier ${ }^{14} \mathrm{C}$-datierter Pollendiagramme kann erstmals die spätglaziale Vegetationsentwicklung im ehemals vergletscherten südlichen Allgäu rekonstruiert werden. Die vorliegenden Pollendiagramme wurden mit anderen Untersuchungen aus dem Alpenvorland und den nördlichen Alpen verglichen und entsprechenden Biozonen zugeordnet. Im Bølling breitet sich in den tieferen Lagen Pinus aus und wird zur dominanten Baumart. In höheren Lagen findet die Ausbreitung verzögert statt. Die Warmphase des Allerøds führt im Vergleich zum Bølling zur Entwicklung dichterer Wälder. Die klimatische Abkühlung der Jüngeren Dryas zeigt sich in einer Auflichtung der Wälder. Desweiteren konnten möglicherweise erstmals im Allgäu die Gerzensee-Schwankung im
\end{abstract} Allerød und das Rammelbeek im Präboreal pollenanalytisch nachgewiesen werden.

Lateglacial vegetation development in the upper Iller region [Allgäu/Bavaria]

Abstract:

Three radiocarbon dated pollen diagrams for the first time reveal the full Lateglacial vegetation development in the formerly glaciated area of the Southern Allgäu. The presented pollen diagrams were compared with other investigations of the Alpine foreland and northern Alps and were assigned to corresponding biozones. In the Bølling Pinus immigrates in lower altitudes and becomes the dominant tree species. In higher altitudes the expansion is delayed. During the Allerød denser pine forests evolve with an understorey of birches. The climatic cooling of the Younger Dryas leads to sparser forests. Furthermore it is likely, that two climatic oscillations were detected pollenanalytically, the Gerzensee-depression in the Allerød and the Rammelbeek-depression in the Preboreal.

Keywords: $\quad$ Vegetation history, Lateglacial, ${ }^{14} \mathrm{C}$-dating, Climatic oscillations, Alps

Addresses of authors: Philipp Stojakowits, Arne Friedmann, Andrea Bull, Arbeitsgruppe Biogeographie, Institut für Geographie, Universität Augsburg, Alter Postweg 118, D-86135 Augsburg, Germany. E-Mails: stojakowits@geo.uni-augsburg.de, friedmann@geo.uni-augsburg.de

\section{Einleitung}

Aus vielen Regionen des deutschen Alpenvorlandes existieren detaillierte pollenanalytische Untersuchungen zum Ablauf der spätglazialen Vegetationsgeschichte. Für die Bodenseeregion mit Oberschwaben wurden z.B. Untersuchungen von Göttlich (1955), Bertsch (1961), LANG (1952, 1962), Müller (1962), MielKe \& Müller (1981), Rösch (1985, 1990) und LeChterbeck (2001) vorgelegt. Die Ammerseegegend wurde von KLEINMANN (1995) bearbeitet. Aus dem südöstlichen Bayern seien die Analysen von SchmeidL (1971), Rausch (1975), Beug (1976) und Voigt (1996) genannt. Obwohl schon seit über 80 Jahren pollenanalytische Forschung im südwestbayerischen Alpenvorland betrieben wird - PAUL \& Ruoff (1932) als Pioniere - ist das Allgäu jedoch bisweilen ein weißer Fleck hinsichtlich der spätglazialen Vegetationsentwicklung. Lediglich von KüsTER (1988) wurde ein Pollendiagramm publiziert, welches das jüngere Spätglazial abdeckt.

Mit der vorliegenden Arbeit wird angestrebt, einen Teil dieser Lücke zu schließen. Es werden zwei Profile aus dem oberen Illertal und eines aus dem angrenzenden Kleinwalsertal vorgestellt. Dabei finden zwei Diagramme aus einer nicht publizierten Dissertation von Dieffenbach-FriEs (1981) ergänzend Berücksichtigung.

\section{Untersuchungsgebiet}

Die vorgestellten Archive liegen in Bayerisch-Schwaben im Landkreis Oberallgäu (Abb. 1). Die Niedersonthofener Seen, zu denen auch der Untere Inselsee (703 m ü. NN) gehört, sind in ein Mosaik aus Jungmoränen und Schichten der Faltenmolasse unweit des morphologischen Alpenrandes eingebettet. Das Profil Moorbad Oberstdorf ist im Illertalboden auf $830 \mathrm{~m}$ ü. NN lokalisiert und von den Gebirgszügen des Helvetikums, der Flyschzone und des Kalkalpins umgeben. Die Vermoorung am Gasthof Bergblick ist auf $1050 \mathrm{~m}$ ü. NN im angrenzenden Kleinwalsertal im Verzahnungsbereich von Flysch und Helvetikum auf einer Hangverebnung gelegen. Weiter südlich schließen ebenfalls kalkalpine Ablagerungen an.

Während der Würmeiszeit wurde das zuvor schon angelegte Illertal vom Illergletscher weiter glazial übertieft. Für 


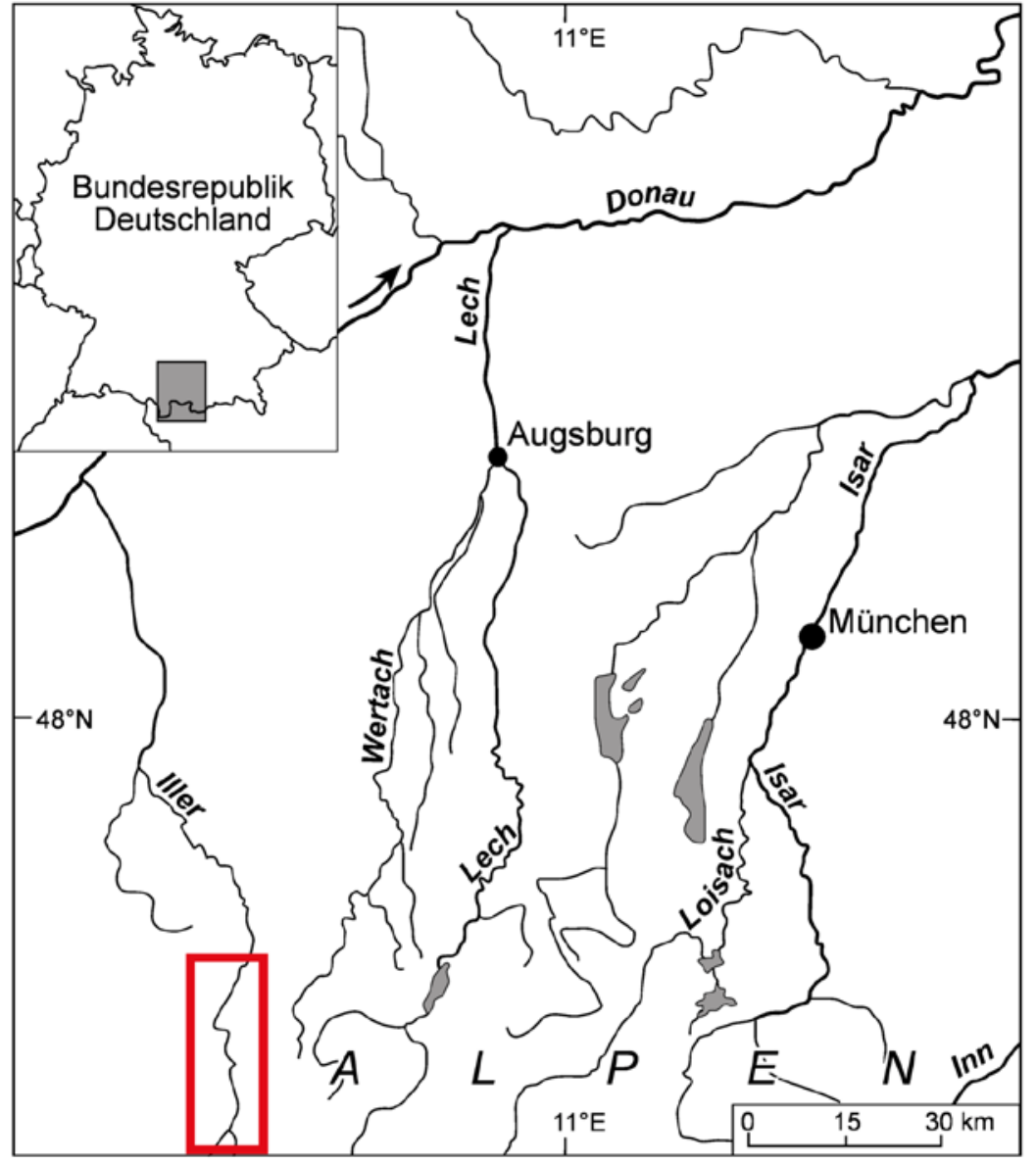

Abb. 1: Lage des Untersuchungsgebietes. Fig. 1: Location of the study area. den Höhepunkt der letzten Eiszeit werden für das Untersuchungsgebiet Eismächtigkeiten von 700 bis $800 \mathrm{~m}$ über dem heutigen Talboden angegeben (BADER \& JERZ 1978: 29). Beim Niedertauen des Illergletschers wurden durch Glazialerosion vertiefte Becken freigegeben. In einem solchen Becken, welches im ausgehenden Würm-Hochglazial durch Toteis vor der Zusedimentierung bewahrt wurde, liegen auch die Niedersonthofener Seen (Jerz 1974: 72). Die Ausschürfung des Beckens wurde wohl durch eine Störungszone mit leichter erodierbaren Molasseschichten begünstigt (JERZ 1974: 81). Im Spätglazial sind der Illergletscher und seine Seitengletscher in die Hochlagen der Allgäuer Alpen zurückgeschmolzen (Scholz 1995: 250). Zur Zeit des Bühl-Stadiums war das Moor am Gasthof Bergblick noch von Gletschereis bedeckt. In diesem und dem darauf folgenden Steinach-Stadium, welche beide jüngst als Phase des frühspätglazialen Eiszerfalls in die Literatur eingingen (REITNER 2007: 82 und Ivy-Ochs et al.: Tab. 1), wurden am Gletschertalrand glazilakustrine Eisstauseeschüttungen in Form von vier Deltaterrassenstufen abgelagert. Die unterste Terrassenstufe ist auf $1050 \mathrm{~m}$ ü. NN ausgebildet (VÖLK 2001: 67).

\section{Methoden}

Am Südwestufer des Unteren Inselsees wurde mit einem Russischen Kammerbohrer ein 7,20 m langer Bohrkern gewonnen. Für die Anreicherung der Pollenkörner im Probenmaterial kamen die gängigen Standardverfahren zur Anwendung (FÆgri \& Iversen 1989, Moore, WebB \& Collinson 1991). Dem Bohrkern wurden alle zwei bis vier cm Proben von einem $\mathrm{cm}^{3}$ aus dem Kerninneren entnommen und mittels Salzsäure-, Natronlauge-, Flusssäure- und Acetolyse-Behandlung aufbereitet. Abschließend wurde mit Hilfe eines Ultraschallbades das Feinmaterial $(<10 \mu)$ ausgesiebt. Um die absolute Pollenkonzentration berechnen zu können, wurden den Proben jeweils zwei Tabletten mit einer definierten Menge Lycopodium-Sporen hinzugefügt (SтоскMARR 1971). Die aufbereiteten Proben wurden unter einem Durchlichtmikroskop mit Kreuztisch bei 400 - bis 1000 -facher Vergrößerung ausgezählt.

Zur sicheren Identifikation der Pollenkörner diente die einschlägige Bestimmungsliteratur (BEUG 1961, FÆGRI \& Iversen 1989, Moore, WebB \& Collinson 1991, Reille 1998). Zusätzlich stand eine umfangreiche Vergleichssammlung rezenter Pollenkörner und Sporen aus Süddeutschland zur Verfügung. Die Nomenklatur der Pollentypen richtet sich nach BEUG (2004).

Die Ergebnisse der Pollenanalysen sind in drei Diagrammen dargestellt (Abb. 2 bis 4). Als Bezugssumme (=100\%) wurde der gesamte Gehölzpollen und Nichtbaumpollen unter Ausschluss der Sporen sowie der lokalen Moor- und Wasserpflanzen gewählt. Ausgeschlossen wurden neben Cyperaceen, Botrychium, Caltha, Lemna, Menyanthes, Myriophyllum, Nuphar, Nymphaea, Parnassia, Polypodiaceae, Potamogeton, Selaginella selaginoides, Sparganium, Sphagnum und Typha. Einige der in den Diagrammen enthaltenen Pollentypen wurden zusammengefasst (z.B. Rosaceae spp.). Die beiden von DiefFenbach-Fries (1981) bearbeiteten Diagramme Moorbad Oberstdorf und Gasthof Bergblick enthielten ursprünglich Cyperaceen in der Bezugssumme, weswegen die- 







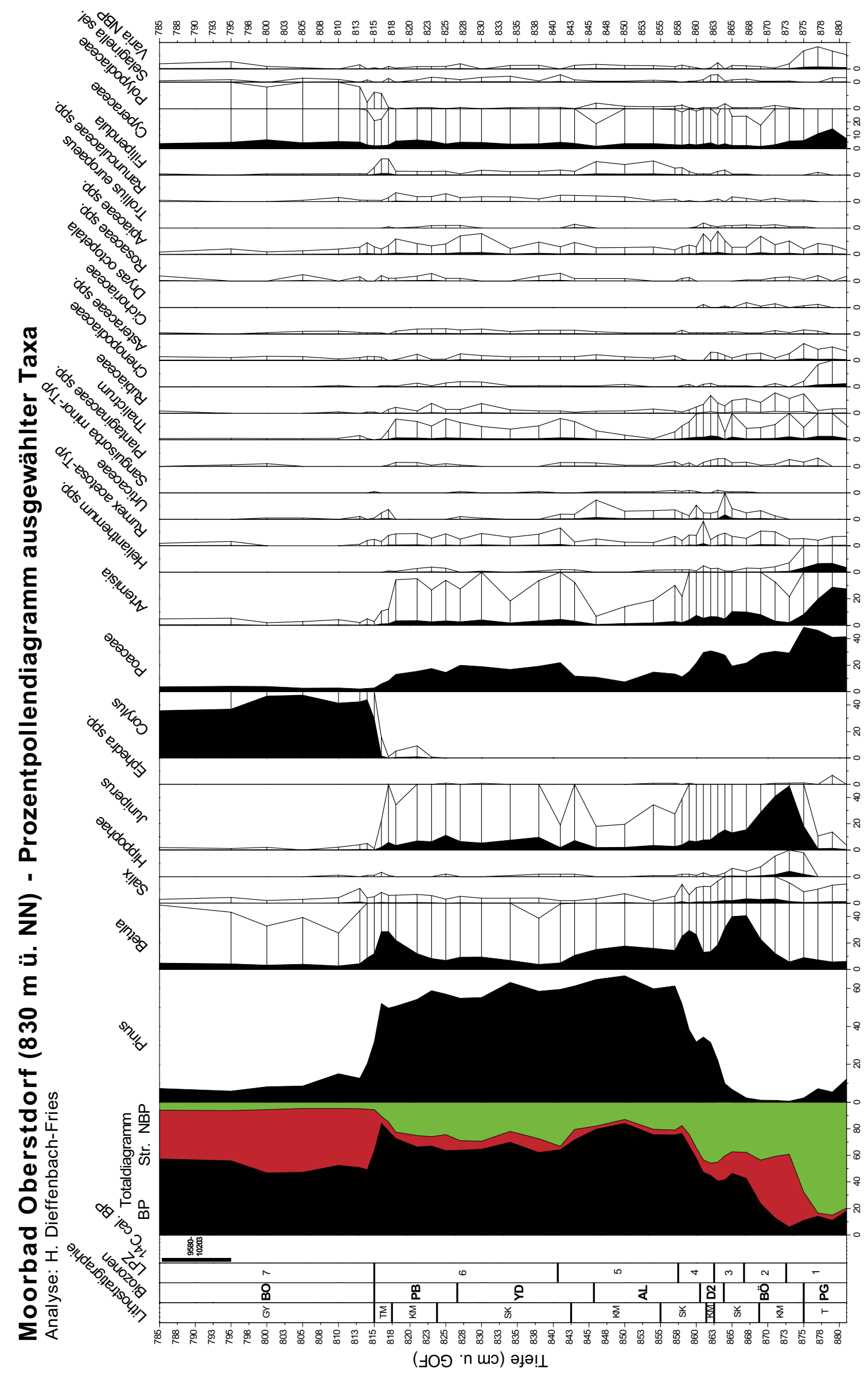

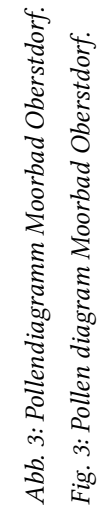




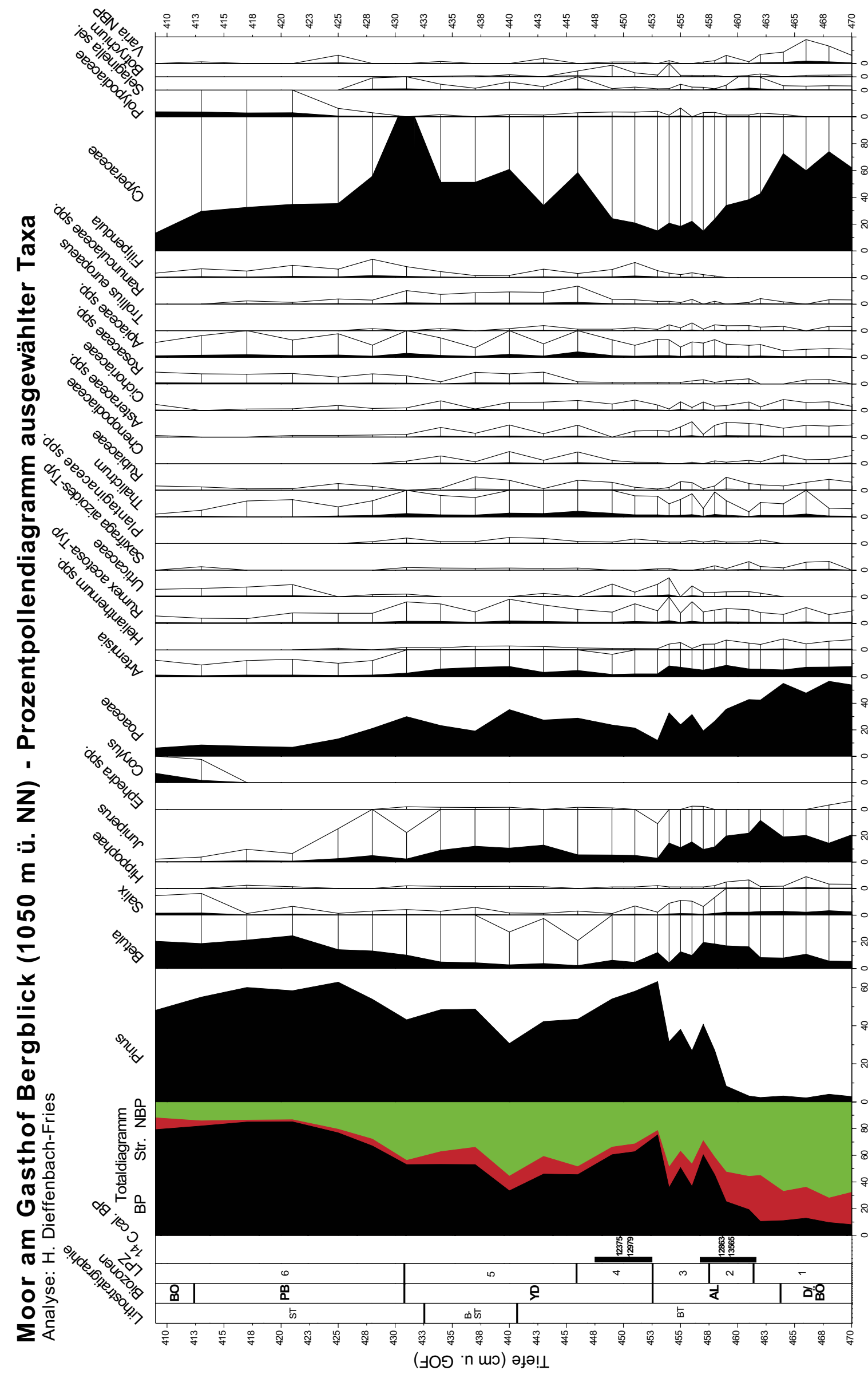


se Profile neu berechnet wurden. Abzüglich der ausgeschlossenen Palynomorphen wurden nahezu durchweg hohe Pollensummen ausgezählt. Im Profil Inselsee wurden abgesehen vom Pleniglazial und der frühen Ältesten Dryas (260-400 Pollenkörner) mindestens 600 Baumpollen- bzw. 1000 terrestrische Pollenkörner pro Probe erfasst. Die Bezugssumme des Profils Oberstdorf beinhaltet 600-900 Pollenkörner, nur nicht im Pleniglazial (300-500). Auf das Profil Bergblick entfallen bis auf eine Probe $(=492)$ jeweils 600-900 Palynomorphen. Die stratigraphischen Einheiten wurden im Gelände bohrbegleitend abgegrenzt. Eine genaue Farb- und Substratansprache erfolgte im Labor, welche durch Makrorestbefunde ergänzt wurde. An vegetationsgeschichtlich markanten Stellen wurde dem Bohrkern Material (bulkproben bei DiEFFENBACH-FRIES 1981) für Datierungszwecke entnommen und mittels AMS ${ }^{14} \mathrm{C}$-Messung datiert. Die kalibrierten ${ }^{14} \mathrm{C}$-Daten wurden mit dem Programm Calib 7.01 (STUIVer \& ReIMER 1993) berechnet und die wahrscheinlichste Alterszeitspanne mit 2 Sigma-Intervall angegeben. Dem Programm lag dabei der Datensatz IntCal13 (REIMER et al. 2013) zugrunde.

\section{Ergebnisse \\ 4.1 Stratigraphie}

Die Stratigraphie ist in den Tabellen 1 bis 3 dargelegt.

\subsection{Datierung}

Die kalibrierten ${ }^{14} \mathrm{C}$-Daten sind in den Tabellen 4 und 5 dargestellt.

\subsection{Lokale Pollenzonen der Profile 4.3.1 Lokale Pollenzonen Diagramm Inselsee}

\section{LPZ 1 (600-588 cm): NBP-Pinus}

Die Nichtbaumpollenanteile und die von Pinus schwanken jeweils zwischen ungefähr 40 und $60 \%$. Die wichtigsten Nichtbaumpollentypen sind nach absteigender Häufigkeit Poaceae, Cyperaceae, Artemisia, Helianthemum und Chenopodiaceae. Bei den Gehölzpollentypen sind Funde von Ephedra erwähnenswert. Insgesamt zeichnet sich der Abschnitt durch eine hohe Anzahl nachgewiesener Taxa aus.

\section{LPZ 2 (588-574 cm): Betula-Poaceae}

Betula und Poaceae sind über mehrere Proben hinweg mit wechselnden Dominanzverhältnissen repräsentiert. Die Pinus-Kurve ist auf ein Minimum abgesunken und nimmt erst gegen Ende des Abschnitts wieder zu. Kurz zuvor erreicht Juniperus einen Gipfel mit $30 \%$ bei zugleich rückläufiger Poaceen-Kurve.

\section{LPZ 3 (574-530 cm): Pinus-Betula}

Die Nachweise der Nichtbaumpollentypen pendeln sich auf sehr niedrigerem Niveau ein und Pinus dominiert fortan das Pollenspektrum. Kurvenschwankungen dieses Taxons fallen mit erhöhten Betula-Werten und einer einmaligen Zunahme der Nichtbaumpollenfunde zusammen.

\section{LPZ 4 (530-498 cm): Pinus-NBP}

Die Pinus-Kurve sinkt ab und schwankt zwischen 60-80\%. Betula ist meist mit unter $10 \%$ Anteil am Pollenspektrum vertreten, nimmt jedoch am Abschnittsende kurzzeitig zu. Bei funiperus und Salix treten kleinere Gipfel auf. Die Nichtbaumpollenwerte liegen bei bis zu $20 \%$, wobei besonders Poaceae und Artemisia hervortreten. Auch viele andere Nichtbaumpollentypen treten wieder deutlicher im Pollenspektrum hervor, wie z.B. Thalictrum, Rubiaceae, Chenopodiaceae, Asteraceae und Cichoriaceae.

\section{LPZ 5 (498-476 cm): Pinus}

Der Nichtbaumpollenanteil geht zurück, der von Pinus nimmt entsprechend zu. Gegen Ende der Pollenzone ist ein Anstieg der Betula-Kurve zu verzeichnen.

\section{LPZ 6 (476-470 cm): Corylus-EMW}

Diese Pollenzone ist durch ansteigende Werte der thermophilen Gehölze gekennzeichnet. Neben dominanter Corylus sind die sogenannten Eichenmischwaldgehölze Ulmus, Tilia und Quercus gehäuft nachgewiesen. Pinus ist nur noch mit Werten um die $20 \%$ vertreten.

\subsubsection{Lokale Pollenzonen Diagramm Oberstdorf}

\section{LPZ 1 (881-873 cm): Poaceae-Artemisia}

In dieser Zone überwiegt der Nichtbaumpollenanteil. Hiervon machen Poaceae 40 bis $50 \%$ aus. Artemisia ist anfangs mit knapp $30 \%$ vertreten, sinkt aber im Verlauf der Pollenzone auf $8 \%$ ab. In etwa gegenläufig dazu verhält sich die funiperus-Kurve am Ende des Abschnitts.

\section{LPZ 2 (873-867 cm): Funiperus-Poaceae-Betula}

funiperus dominiert zwar durchweg den Polleninhalt, dessen Werte fallen jedoch von 49 auf $28 \%$ ab bei zugleich steigenden Betula-Gehalten. Die Poaceen-Anteile sind bei um die $30 \%$ stabil.

\section{LPZ 3 (867-863 cm): Betula-Poaceae-Juniperus}

In dieser Zone sind die Dominanzverhältnisse umgekehrt. Betula ist mit 31 bis $40 \%$ am häufigsten nachgewiesen. Funiperus ist mit etwa $15 \%$ deutlich seltener geworden. Poaceae erreichen 19 bis $28 \%$.

\section{LPZ 4 (863-858 cm): Pinus-Poaceae-Betula}

Die Pinus-Werte liegen anfangs bei rund $20 \%$ und nehmen im Verlauf des Abschnitts bei kurzzeitiger Kodominanz mit Poaceen zu. Deren Nachweise gehen zum Zonenende hin zurück und neben Pinus ist Betula stärker im Pollenspektrum repräsentiert.

\section{LPZ 5 (858-841 cm): Pinus-Betula-Poaceae}

Pinus ist zum dominanten Taxon geworden bei Anteilen von 52 bis $67 \%$. Poaceae sind mit Werten um die $10 \%$ vertreten und die Betula-Nachweise fallen von anfänglich $25 \%$ auf etwa $15 \%$ ab.

\section{LPZ 6 (841-815 cm): Pinus-Poaceae}

Das Pollenspektrum wird weiterhin von Pinus dominiert. Jedoch ist Betula - vom Abschnittsende einmal abgesehen - deutlich seltener und die Poaceen-Anteile sind deutlich erhöht. Ebenso sind andere Nichtbaumpollentypen (z.B. Artemisia, Thalictrum) wie auch funiperus häufiger. 
Tab. 1: Stratigraphie der Kernbohrung Unterer Inselsee.

Tab. 1: Stratigraphy of the Unterer Inselsee core.

\begin{tabular}{|c|l|}
\hline $\begin{array}{c}\text { Tiefe } \\
\text { (in cm u. GOF) }\end{array}$ & \multicolumn{1}{c|}{ Sediment/ Sedentat } \\
\hline $470-476$ & Seekreide (gräulich gelb, Conchylien) \\
\hline $476-483$ & Seekreide (grau bis ockerfarben, Conchylien) \\
\hline $483-498$ & Seekreide (ockerfarben, Conchylien) \\
\hline $498-524$ & Seekreide (gräulich gelb) \\
\hline $524-529$ & Seekreide (dunkelgräulich gelb) \\
\hline $529-567$ & Seekreide (ockerfarben) \\
\hline $567-583$ & Seekreide (grau) \\
\hline $583-588$ & Carbonatreicher Beckenton bis tonige Seekreide \\
\hline $588-720$ & Carbonatreicher Beckenton \\
\hline
\end{tabular}

Tab. 2: Stratigraphie der Kernbohrung Moorbad Oberstdorf.

Tab. 2: Stratigraphy of the Moorbad Oberstdorf core.

\begin{tabular}{|c|l|}
\hline $\begin{array}{c}\text { Tiefe } \\
\text { (in cm u. GOF) }\end{array}$ & \multicolumn{1}{c|}{ Sediment/ Sedentat } \\
\hline $785-815$ & Gyttja \\
\hline $815-818$ & Carbonatreiche Torfmudde (Gyttja) \\
\hline $818-824$ & Tonreiche Kalkmudde \\
\hline $824-843$ & Tonreiche Seekreide (sehr wenig Conchylien); Pinus-Spaltöffnungen \\
\hline $843-855$ & Kalkmudde (Conchylien); Pinus-Spaltöffnungen; 1 Hippophä̈-Samen \\
\hline $855-862$ & Seekreide (Conchylien); Pinus-Spaltöffnungen \\
\hline $862-863$ & Kalkmudde \\
\hline $863-869$ & Seekreide (Conchylien) \\
\hline $869-875$ & Kalkmudde (wenig Conchylien); Juniperus-Spaltöffnungen \\
\hline $875-882$ & Carbonatreicher Seeton bis tonreiche Seekreide \\
\hline
\end{tabular}

Tab. 3: Stratigraphie der Kernbohrung Gasthof Bergblick.

Tab. 3: Stratigraphy of the Gasthof Bergblick core.

\begin{tabular}{|c|l|}
\hline $\begin{array}{c}\text { Tiefe } \\
\text { (in cm u. GOF) }\end{array}$ & \multicolumn{1}{c|}{ Sediment/ Sedentat } \\
\hline $409-433$ & $\begin{array}{l}\text { Seggentorf mit Carex limosa, Carex flava agg., Carex pallescens, } \\
\text { Potentilla erecta, Betula alba s.l.-Nüsse }\end{array}$ \\
\hline $433-441$ & Braunmoos-Seggentorf mit Calliergon trifarium, Carex limosa \\
\hline $441-468$ & Braunmoostorf mit Calliergon trifarium \\
\hline $468-470$ & $\begin{array}{l}\text { Tonhaltiger Braunmoostorf mit Drepanocladus revolvens, Calliergon } \\
\text { trifarium, Eleocharis cf. Palustris }\end{array}$ \\
\hline
\end{tabular}




\section{LPZ 7 (815-785 cm): Corylus-EMW}

Der Polleninhalt wird von Corlyus mit Werten zwischen 30 und $47 \%$ dominiert, gefolgt von Ulmus mit 12 bis $20 \%$. Andere nicht im Pollendiagramm dargestellte Laubgehölze (Fraxinus, Quercus, Tilia cordata, Tilia platyphyllos) überschreiten im Zonenverlauf $5 \%$ und die Pinus-Nachweise gehen von 30 auf $7 \%$ zurück. Nichtbaumpollentypen sind nur noch sehr spärlich vorhanden.

\subsubsection{Lokale Pollenzonen Diagramm Bergblick}

LPZ 1 (470-462 cm): Cyperaceae-Poaceae-Funiperus Der Pollengehalt wird von Cyperaceen, Poaceen und funiperus bestimmt, wobei die Cyperaceen-Nachweise zwischen 60 und knapp $75 \%$ liegen. Poaceen sind mit Werten um die $50 \%$ vertreten und funiperus erreicht zwischen 15 und $20 \%$. Am nächst häufigen treten Artemisia und Betula hervor.

\section{LPZ 2 (462-458 cm): Poaceae-Cyperaceae-funiperus}

Mit dem Beginn der Zone fällt ein Funiperus-Gipfel zusammen. In deren Verlauf gehen die Anteile von Poaceen, Cyperaceen und funiperus zurück, während die Werte von Betula und verzögert auch von Pinus zunehmen.

LPZ 3 (458-453 cm): Pinus-Poaceae-Cyperaceae-Betula Im Pollenspektrum dominieren Pinus und Poaceae bei schwankenden Verhältnissen. Mit absteigender Häufigkeit folgen Cyperaceae, Betula und Juniperus. Die ArtemisiaFunde sind gegenüber den vorangegangenen Abschnitten nicht signifikant verändert.

\section{LPZ 4 (453-446 cm): Pinus-Poaceae-Cyperaceae}

Diese Pollenzone ist durch die Vorherrschaft von Pinus gekennzeichnet. Poaceae und Cyperaceae sind mit steigenden Kurvenverläufen auf je $24 \%$ repräsentiert. Die funiperusund auch Betula-Nachweise sind seltener geworden.

\section{LPZ 5 (446-431 cm): Pinus-Cyperaceae-Poaceae}

Der Pollengehalt wird durch Pinus und Cyperaceae bestimmt, wobei Pinus an Bedeutung eingebüßt hat. Entsprechend treten Poaceae, funiperus und Artemisia stärker hervor. Zudem häufen sich die Nachweise verschiedener Nichtbaumpollentypen (z.B. Thalictrum und Apiaceae).

\section{LPZ 6 (431-409 cm): Pinus-Cyperaceae-Betula}

Am Beginn des Abschnitts ist ein Cyperaceen-Maximum mit $115 \%$ Anteil bezogen auf die zugrunde gelegte Pollensumme zu verzeichnen. Die Pinus-Kurve pendelt sich auf deutlich höherem Niveau mit Werten zwischen 48 und $62 \%$ ein. Betula gewinnt ebenfalls an Bedeutung im Polleninhalt. In der ersten Hälfte der Zone sinken die Cyperaceen- und PoaceenAnteile deutlich ab und stabilisieren sich nachfolgend.

\section{Diskussion}

\subsection{Vegetationsgeschichtlicher Ablauf und die} Zuordnung zu biostratigraphischen Phasen

\subsubsection{Vegetationsgeschichte Diagramm Unterer} Inselsee

Im tiefsten Diagrammabschnitt $(600-591 \mathrm{~cm})$, der in das ausgehende Würm-Hochglazial gestellt wird, dominiert der
Gräser- und Kräuterpollenanteil. Dieser enthält relativ hohe Anteile an Pionierpflanzen und Heliophyten. Als häufigste Nichtbaumpollen finden sich Poaceae, Cyperaceae, Artemisia, Helianthemum, Chenopodiaceae, Cichoriaceae, Thalictrum, Brassicaceae und Asteraceae. Desweiteren sind Ephedra, Hippophaë und Salix nachweisbar.

Die hohen Pinus-Werte (40-60\%) sind unter Offenlandbedingungen als Fernflug zu interpretieren (LANG 1994). Es ist von einer baumlosen Tundren-Vegetation auszugehen, die eine nicht geschlossene, lückenhafte Vegetationsdecke (sehr geringe Pollenkonzentration!) aus Pionierpflanzen und Steppenelementen aufweist. Je nach Definition wird in der Schweiz diese Phase ebenfalls in das ausgehende Pleniglazial oder davon abweichend in den frühesten Teil der Ältesten Dryas gestellt (AmMANN et al. 1994: 121).

Der nächste Diagrammabschnitt (591-578 cm) wird der Ältesten Dryas zugeordnet. Mit deren Einsetzen kommt es zu einer Erhöhung der Betula-, Hippophä̈-, Funiperus- und Salix-Prozentwerte sowie der Pollenkonzentration. Gleichzeitig gehen die Fernflugeinträge von Pinus $(<8 \%)$ und die Cyperaceen- und Kräuterpollenanteile (z.B. Artemisia) deutlich zurück. Dies deutet auf die Etablierung einer Strauchvegetation hin, obwohl der Nichtbaumpollenanteil jedoch weiterhin hoch bleibt, da Poaceen an Bedeutung gewinnen. Die Betula-Kurve schwankt zwischen 30 und $40 \%$, was auf das Näherrücken der Arealgrenze hinweist, aber auch schon die Möglichkeit des vereinzelten Auftretens anderer BetulaArten (außer Betula nana) einschließt. Verglichen mit den palynologischen Befunden aus der Schweiz (Ammann et al. 1994: 121) könnte es sich hierbei um die sogenannte Betula nana-Subzone innerhalb der Ältesten Dryas handeln.

Mit Beginn des Bølling-Interstadials $(578-568 \mathrm{~cm})$ tritt der für das Alpenvorland charakteristische funiperus-Gipfel auf. Dieser liegt bei $33 \%$ der Bezugssumme. Bei entsprechend dichterer Auszählung könnten wohl Proben mit noch höheren Funiperus-Anteilen erfasst werden. So liegt das $\mathrm{Fu}$ niperus-Maximum am Schleinsee bei über $70 \%$ (MIELKE $\mho$ MüLLER 1981: Fig. 6.9). Nachfolgend kommt es zu einer deutlichen Zunahme der Pinus-Werte, was das Heranrücken der Arealgrenze anzeigt. Im Laufe dieses vegetationsgeschichtlichen Abschnitts wandert Pinus schließlich ein und die Offenlandanteile gehen leicht zurück. Dabei verlieren insbesondere Poaceen und Artemisia an Bedeutung. Der Rückgang der Betula-Kurve von 30 auf $10 \%$ ist relativ zu sehen, da er auf die Zunahme der Pinus-Kurve zurückzuführen ist. Gegen Ende des Bøllings bestimmt ein sehr lichter Kiefernwald mit mosaikartigen Offenlandbereichen das Landschaftsbild. Den Kiefernwäldern ist untergeordnet Betula beigemischt. Die Abgrenzung dieses vegetationsgeschichtlichen Abschnitts zum Liegenden hin ist allerdings nicht gesichert. Typischerweise ist für das frühe Bølling im südlichen Mitteleuropa ein ausgeprägtes funiperus-Maximum zu verzeichnen, so im süddeutschen Alpenvorland (vgl. z.B. BERTSCH 1961, LECHTERBECK 2001, MiELKE \& MülLER 1981 SCHMEIDL 1971) und in der Schweiz (vgl. z.B. AmmanN \& LotTer 1989, AmmANN et al. 2013, LotTER et al. 1992). Mit den erhöhten JuniperusNachweisen im Verein ist auch Hippophaë verstärkt im Pollenspektrum repräsentiert, weswegen das frühe Bølling von BerTsch (1961: 257) sowie von AmMAnN et al. (1994: 121) und Ammann et al. (2013: Fig. 7) als Funiperus-Hippophä̈Strauchphase bezeichnet wird. Folglich könnte im vorlie- 
Tab. 4: AMS ${ }^{14} C$-Daten aus dem Bohrkern Unterer Inselsee. Datierung durch Dr. W. A. Keller am Geogr. Institut der Univ. Zürich und dem Institut für Teilchenphysik der ETH-Hönggerberg (2003) sowie durch Dr. A. Scharf am AMS-Labor Erlangen des Physikalischen Instituts der Univ. Erlangen-N. (2011). Kalibriert (für cal. BP-Alter) mit Calib 7.01.

Tab. 4: AMS ${ }^{14} C$-dates from the Unterer Inselsee core. Dated by Dr. W. A. Keller Geogr. Department Univ. Zürich and Department of Physics ETH-Hönggerberg (2003) and Dr. A. Scharf AMS-laboratory Erlangen of the Physics Department Univ. Erlangen-Nürnberg (2011). Calibrated (for cal. BP-ages) with Calib 7.01.

\begin{tabular}{|c|c|c|c|c|}
\hline Labor-Nr. & $\begin{array}{c}\text { Tiefe } \\
\text { (cm u. GOF) }\end{array}$ & Probenmaterial & $\begin{array}{c}\text { konv. }{ }^{\mathbf{1 4} \mathbf{C}-} \\
\text { Alter BP }\end{array}$ & $\begin{array}{c}\text { Kalib.-Alter } \\
\text { cal. BP (2 } \boldsymbol{\sigma})\end{array}$ \\
\hline UZ-4831 & 571 & Holz & $12210 \pm 95$ & $13776-14565$ \\
\hline UZ-4830 & 550 & Holz & $11760 \pm 90$ & $13431-13763$ \\
\hline UZ-4887 & 539 & Holz & $11380 \pm 90$ & $13074-13404$ \\
\hline UZ-4886 & 531 & Kiefernnadel & $10610 \pm 95$ & $12376-12729$ \\
\hline UZ-4885 & 517 & Holz & $10470 \pm 85$ & $12076-12682$ \\
\hline Erl-16266 & 474 & Blattreste & $9306 \pm 71$ & $10278-10681$ \\
\hline
\end{tabular}

Tab. 5: ${ }^{14} \mathrm{C}$-Daten aus den Bohrkernen Moorbad Oberstdorf und Gasthof Bergblick. Datierung durch Dr. M. Geyh. Kalibriert (für cal. BPAlter) mit Calib 7.01 .

Tab. 5: ${ }^{14} \mathrm{C}$-dates from the Moorbad Oberstdorf and Gasthof Bergblick. Dated by Dr. M. Geyh. Calibrated (for cal. BP-ages) with Calib 7.01.

\begin{tabular}{|c|c|c|c|c|}
\hline Labor-Nr. & $\begin{array}{c}\text { Tiefe } \\
\text { (cm u. GOF) }\end{array}$ & $\begin{array}{c}\text { Proben- } \\
\text { material }\end{array}$ & $\begin{array}{c}\text { konv. }{ }^{\mathbf{1 4}} \mathbf{C}- \\
\text { Alter BP }\end{array}$ & $\begin{array}{c}\text { Kalib.-Alter } \\
\text { cal. BP (2 } \boldsymbol{\sigma})\end{array}$ \\
\hline Hv 10257 (Oberstdorf) & $785-795$ & Torfbulk & $8845 \pm 125$ & $9580-10203$ \\
\hline Hv 9986 (Bergblick) & $457-462$ & Torfbulk & $11390 \pm 175$ & $12863-13565$ \\
\hline Hv 10474 (Bergblick) & $448-453$ & Torfbulk & $10725 \pm 145$ & $12375-12979$ \\
\hline
\end{tabular}

genden Diagramm der Beginn des Bøllings auch schon bei $591 \mathrm{~cm}$ gezogen werden. Somit würde der funiperus-Gipfel etwa ins mittlere Bølling datieren, was mit den palynologischen Befunden aus dem österreichischen Vorarlberg von vAN Mourik et al. (2013: Fig. 6) übereinstimmen würde. Im Gegensatz dazu werden in anderen Profilen aus Österreich Juniperus-Maxima mitunter in die ausgehende Älteste Dryas gestellt (vgl. BoRTENSCHLAGER 1984), bei WAHLMÜLLER (1985) an die Wende Älteste Dryas - Bølling.

Der kurze Abschnitt der Älteren Dryas $(568-564 \mathrm{~cm})$ zeichnet sich im Pollendiagramm nur schwach ab. Eine geringe Zunahme der Betula- und Nichtbaumpollen-Werte steht einem Einschnitt in der Pinus-Kurve gegenüber. Wie BLUDAU (1985: 156) anführt, könnte die Klimaverschlechterung der Älteren Dryas eine verringerte Blühfähigkeit von Pinus bewirkt haben. Für die Vegetationszusammensetzung lassen sich daraus jedoch keine Veränderungen gegenüber dem Bolling ableiten. Eine Verminderung der Pollenkonzentration ist ebenfalls nicht feststellbar. Lediglich gegen Ende des Bolling ist ein Einschnitt in der Pollendichte zu verzeichnen.
Mit dem Übergang zum Allerød $(564-530 \mathrm{~cm})$ nehmen die Pinus-Werte auf bis zu $90 \% \mathrm{zu}$ und die NBP-Anteile gehen deutlich zurück. Es ist von einem lichten Kiefernwald mit standortsbedingt stärkeren Birkenvorkommen auszugehen. Im mittleren Allerød ist vermutlich die von EICHER \& Siegenthaler (1976) beschriebene Gerzensee-Schwankung erfasst. Deren Einsetzen konnte auf 13431-13763 cal. BP datiert werden. Im Pollendiagramm zeigt sich ein Rückgang der Pinus-Werte bei gleichzeitigem Anstieg der Betula-, Salix- und NBP-Kurven. Diese Veränderungen deuten auf eine kurzzeitige Auflichtung der Kiefernwälder und erhöhte Offenlandanteile hin. Im weiteren Allerød gehen die NBP-Werte wieder zurück. Die Auswirkungen der Klimaschwankung finden aber laut LotTER et al. (1992: 187) palynologisch keinen Niederschlag. Bei der von WICK (2000: Fig. 1) durchgeführten Untersuchung am locus typicus des Gerzensees sind während dieser Klimaoszillation die Pinus-Werte erhöht bei zugleich niedrigeren Betula-Anteilen. Die jüngst dort besorgte Pollenanalyse weist keine deutlichen Signale in den Kurvenverläufen von Betula und Pinus wie auch den Nichtbaumpollennachweisen auf (AMmANn et al. 2013: Fig. 2). 
Mit dem Beginn der Jüngeren Dryas (530-498 cm) nehmen die NBP-Anteile (bis zu $20 \%$ ) sowie die Salix-, Betulaund Funiperus-Werte zu. Die Pinus-Kurve geht zurück und schwankt zwischen 60 und $80 \%$. Bei den Nichtbaumpollen sind wieder verstärkt Artemisia, Helianthemum, Chenopodiaceae und Thalictrum nachweisbar. Die Pollendichte pendelt sich gegenüber dem Allerød auf einem deutlich niedrigeren Niveau ein. Für den Abschnitt der Jüngeren Dryas ist somit von einer markanten Verschiebung der Wald-Offenlandanteile auszugehen. Ebenfalls ändert sich auch die Farbe der ausgefällten Seekreiden. Die ockerfarbenen Seekreiden des Allerøds gehen in dunkelgraue bis graue über.

Die Grenze zwischen Spätglazial und Holozän ist bei 498 $\mathrm{cm} \mathrm{zu}$ ziehen. Mit Einsetzen des Präboreals (498-476 cm u. GOF) kommt es zu einem starken Rückgang der NBP-Werte und einem Anstieg der Pinus-Kurve auf $90 \%$. Die geschlossene Corylus-Kurve setzt ein und zeigt das Näherrücken der Arealgrenze an. Die Pollenkonzentration steigt auf die höchsten im Diagramm erfassten Werte. Mit der Erwärmung kommt es zur Ausbildung relativ geschlossener Kiefernwälder. Pionier- und Steppenelemente werden entsprechend zurückgedrängt. Bei $480 \mathrm{~cm}$ ist eine kurze Klimaverschlechterung erfasst. Hierbei handelt es sich vermutlich um die sog. Rammelbeek- bzw. Schlaten-Schwankung, welche von BEHRE (1978: Tab. 1 und Tab. 2) ins mittlere Präboreal gestellt wurde. Diese zeichnet sich im Pollendiagramm durch einen deutlichen Einbruch in der Pinus-Kurve und einen starken Anstieg der Betula-Werte ab. Untergeordnet sind auch die Nachweise von Salix, Artemisia, Cichoriaceae und Brassicaceae gehäuft. Das von Rösch (1990: 49) bearbeitete Durchenbergried enthält ebenfalls schwache palynologische Hinweise, welche auf diese Klimaschwankung hindeuten. Zudem ist dort der Glühverlust erniedrigt. Jedoch legen jüngere Untersuchungen (vgl. z.B. Bos et al. 2007) nahe, dass die Rammelbeek-Phase in Mitteleuropa keineswegs synchron verlaufen ist und entsprechende regionale Unterschiede $\mathrm{zu}$ konstatieren sind. Oft zeigt sich von palynologischer Seite kein deutlicher Niederschlag im Gegensatz zu den $\delta{ }^{18} \mathrm{O}-$ Schwankungen (vgl. Wick 2000). Die im Diagramm Inselsee erhöhten Betula-Werte könnten nämlich auch durch eine Seespiegelschwankung mit eventuell einhergehendem Hiatus verursacht worden sein, wie es KLEINMANN (1995: z.B. 320 und 329) in Profilen des Ammersees aufzeigen konnte.

\subsubsection{Vegetationsgeschichte Diagramm Oberstdorf}

Das Profil setzt im Pleniglazial $(881-875 \mathrm{~cm})$ ein. Das Pollenspektrum unterscheidet sich von demjenigen des Unteren Inselsees durch geringere Pinus-Fernflugeinträge bei zugleich höheren Poaceen- und Artemisia-Anteilen. Helianthemum ist in etwa gleich stark repräsentiert. Im Liegenden des Beckentons untersuchte tonig-schluffige Sedimente waren absolut pollenleer, im Hangenden liegt ein Hiatus vor.

Das Bølling $(875-864 \mathrm{~cm})$ beginnt mit einer funiperusHippophä̈-Strauchphase, in der vor allem Artemisia und Helianthemum entscheidend an Verbreitung verlieren. Darauf folgt die zögerliche und lückenhafte Wiederbewaldung mit Betula-Exemplaren.

Die nachfolgende Ältere Dryas $(864-861 \mathrm{~cm})$ ist durch höhere NBP-Werte repräsentiert. Infolge der Klimadepression kommt es zu einer Stillstandsphase der Waldausbreitung.
Eine stärkere Entwaldung ist aber nicht anzunehmen.

Im Allerød (861-846 cm) vollzieht sich die flächendeckende Wiederbewaldung der Tallagen. Die erfolgte Einwanderung von Pinus wird durch bei $857 \mathrm{~cm}$ identifizierten Stomata belegt. Die hohen NBP-Werte im Vergleich zum Profil Inselsee sind auf eingewehte Pollenkörner aus den umgebenden Gebirgszügen zurückzuführen.

Die Jüngere Dryas $(846-827 \mathrm{~cm}$ ) führt zu einer Auflichtung der Wälder. Zum Höhepunkt dieser Kaltphase beträgt der Baumpollenanteil nur noch 67 \%. Damit ist der Grad der Auflichtung deutlich größer wie im Profil Inselsee. Vergleichsweise treten insbesondere rasenanzeigende Taxa wie Poaceae und Thalictrum stärker auf, was nicht nur mit den lichter werdenden Wäldern in Zusammenhang steht, sondern auch eine Absenkung der Waldgrenze im Gebiet widerspiegelt.

\subsubsection{Vegetationsgeschichte Diagramm Bergblick}

Der basale Diagrammabschnitt $(470-464 \mathrm{~cm})$ dürfte wohl dem Bølling und der Älteren Dryas zuzurechnen sein. Die funiperus-Werte um $20 \%$ bei gleichzeitig geringen BPWerten deuten lockere Strauchgesellschaften an. Daneben ist von gehölzfreien Pioniergesellschaften auszugehen, die sich u.a. aus Offenlandzeigern wie Helianthemum, Saxifraga aizoides-Typ und Selaginella selaginoides zusammensetzen. Gemäß den Untersuchungen von WAHLmüLlER (1985: Beilagendiagramme Mieminger See und Seefelder See) könnte dieser Abschnitt auch in die Älteste Dryas gestellt werden. In Konsequenz müsste dann der hier ins Allerød (464-453 $\mathrm{cm}$ ) fallende kleinere funiperus-Gipfel den Übergang zum Bølling markieren. Folglich wäre die ein allerødzeitliches Alter indizierende ${ }^{14} \mathrm{C}$-Datierung als falsch anzusehen. Die Verfasser wie auch DiEFFENBACH-FrIEs (1981: 68) gehen aber von einer erst allerødzeitlichen Wiederbewaldung aus, welche durch eine birkenreiche Phase initiiert wird. Nachdem Pinus eingewandert war, treten im weiteren Allerød zwei markante Schwankungen in den Kurven von Pinus und Betula bei gleichzeitigem Anstieg von funiperus, Poaceen und anderen Pollentypen auf. Hierbei könnte es sich zum einen um die Gerzensee-Schwankung und zum anderen um die Auswirkungen des Laacher-See-Ausbruchs handeln, obwohl Tuffablagerungen nicht vorhanden sind.

Die Jüngere Dryas $(453-431 \mathrm{~cm})$ bedingt ein deutliches Lichterwerden der Pinus-Bestände (bis zu $56 \%$ NBP) in dieser Höhenlage, darüber werden Wald- und Baumgrenze um etwa $200 \mathrm{~m}$ herabgedrückt, wenn man die von BuRGA \& PERRET (1998: 629) ausgewerteten Makrorestbefunde aus vergleichbaren Gebirgsräumen der Schweizer Voralpen zugrunde legt. Gemäß IRMLER \& Scholz (2005: 97 und Fig. 5) könnte die errechnete Schneegrenzdepression während dieses Kälterückschlags in der Allgäuer Nagelfluhkette auf 1400 $m$ ü. NN herabgereicht haben. Für diese rund 20 Kilometer weiter südlich gelegene Lokalität ergibt sich infolge des Massenerhebungseffekts eine ungefähre Schneegrenzlage von $1700 \mathrm{~m}$ ü. NN. Die infolge dessen deutlich erniedrigte Waldgrenze dürfte sich auch in der Zunahme der CyperaceenAnteile zeigen. Ein nicht quantifizierbarer Teil der gezählten Cyperaceen-Pollenkörner entstammt sicherlich einem damaligen Pendant heutiger Polsterseggenrasen (Caricetum firmae Lüdi 1921). 
Tab. 6: Gegenüberstellung der Warvenchronologien und zugehörigen Biozonen vom Meerfelder Maar (LITT \& STEBICH 1999, LITT et al. 2001) und vom Steißlinger See (EUSTERHUES et al. 2002) sowie der jüngst veröffentlichten Altersangaben aus der Schweiz (AmMANN et al. 2013).

Tab. 6: Comparison of the varve chronologies and related biozones from Meerfelder Maar (LITT \& STEBICH 1999, LITT et al. 2001) and from Steisslinger See (EUSTERHUES et al. 2002) and most recent published dates from Switzerland (AMMANN et al. 2013).

\begin{tabular}{|c|c|c|c|c|c|}
\hline \multicolumn{2}{|c|}{ Meerfelder Maar } & \multicolumn{2}{|c|}{ Steißlinger See } & \multicolumn{2}{|c|}{ Schweiz } \\
\hline $\begin{array}{l}\text { Warvenjahre } \\
\text { vor heute }\end{array}$ & Biozonen & $\begin{array}{l}\text { Warvenjahre } \\
\text { vor heute }\end{array}$ & Biozonen & $\begin{array}{l}\text { Alter kalibriert } \\
\text { vor heute }\end{array}$ & Biozonen \\
\hline $11590-12680$ & Jüngere Dryas & $11580-12690$ & Jüngere Dryas & ca. $11570-12710$ & Jüngere Dryas \\
\hline $12680-13350$ & Allerød & \multirow[t]{3}{*}{$12690-13787$} & \multirow[t]{3}{*}{ Allerød } & \multirow[t]{3}{*}{ ca. $12710-13835$} & \multirow[t]{3}{*}{ Allerød } \\
\hline $13350-13540$ & Ältere Dryas & & & & \\
\hline $13540-13780^{*}$ & Bølling & & & & \\
\hline $13780-13910$ & Älteste Dryas & \multirow[t]{2}{*}{$13787-14700^{* *}$} & \multirow[t]{2}{*}{ Bølling } & \multirow[t]{2}{*}{ ca. $13835-14665$} & \multirow[t]{2}{*}{ Bølling } \\
\hline $\begin{array}{l}13910-\text { ca. } \\
14560 \\
\text { (interpoliert) }\end{array}$ & Meiendorf & & & & \\
\hline nicht gewarvt & Pleniglazial & $14700-15500$ & Älteste Dryas & ca. $14665-15676^{* * *}$ & Älteste Dryas \\
\hline
\end{tabular}

*= Hiatus von 110 Jahren (vgl. STEBICH et al. 2009: Fig. 5), wodurch sich die älteren Biozonen analog nach hinten verschieben

**= im ausgehenden Bølling wäre eine Ausgliederung durch einen von kleinen Kurvengipfeln von Poaceen,

Helianthemum und Thalictrum gekennzeichneten Abschnitt möglich

$* * *=$ letztes Drittel der Ältesten Dryas (AMMANN et al. 2013: Fig. 7)

\subsection{Gestaffelte Pinus-Ausbreitung und sich entwickelnde Höhenstufung im oberen Illertal}

Sofern die besprochenen Diagramme korrekt abgegrenzt wurden und keine falschen Altersdatierungen vorliegen, ist von einer zeitlich verzögerten Pinus-Ausbreitung im oberen Illertal auszugehen, welche vom Alpenvorland her erfolgte. Makrorestbefunden zufolge fällt die Wiedereinwanderung von Pinus sylvestris im süddeutschen Alpenvorland in das späte Bølling (Mielke \& MüLLER 1981: Fig. 6.9), wobei LANG (1952: Fig. 3) den ersten Nadelfund schon im frühen bis mittleren Bølling erbracht hat. Für die Lokalität Inselsee ist gemäß der Pollenstratigraphie eine im späten Bølling erfolgte Pinus-Immigration zu konstatieren, welche durch die ${ }^{14} \mathrm{C}$-Datierung bei $571 \mathrm{~cm}$ mit einer Alterszeitspanne von 13776-14565 cal. BP nachgezeichnet wird. Im 25 Kilometer weiter südlich und 130 Meter höher gelegenen Raum Oberstdorf ereignet sich die Pinus-Einwanderung erst in der Älteren Dryas oder gar beginnenden Allerød, je nachdem welcher Prozentschwellenwert herangezogen wird. Die in einem südwestlich anschließenden Seitental befindliche Vermoorung am Gasthof Bergblick auf 1050 m ü. NN zeigt eine mittelallerødzeitliche Ansiedlung von Pinus an. Die an einer $5 \mathrm{~cm}$ mächtigen Torfbulkprobe niedergebrachte Radiokarbondatierung mit einem Alter von 12863-13565 cal. BP umgrenzt grob das Einwanderungsgeschehen. Ob im Allerød schon eine Höhenstufung mit Pinus sylvestris und darüber befindlicher Pinus mugo existiert hat, ist wie in der Schweiz (Burga \& PERret 1998: 402) mangels aussagekräftiger Makrorestbelege nicht gesichert. So sind bei den von DiefFENBACH-Fries (1981: Tab. 21 und 22) an der Lokalität Bergblick durchgeführten Makrorestanalysen erst ab dem
Frühholozän nicht näher bestimmte Pinus-Großrestfunde belegt. Auch muss die Frage nach einer Beteiligung von $\mathrm{Pi}^{-}$ nus cembra am Aufbau der Wald- und Baumgrenze offen bleiben, da eine Trennung in einen Pinus sylvestris-Typ und einen Pinus cembra-Typ in den vorliegenden Analysen nicht vorgenommen wurde. Im östlich angrenzenden Ammergebirge sind jedoch gemäß des Pollenbefundes frühholozäne Arvenbestände im Waldgrenzbereich zu vermuten (ОвIDOWICZ \& SchoBER 1985: 157). Analog zu den Ausführungen von BurgA \& PERRet (1998: 628) die Schweizer Voralpen betreffend könnte in den Allgäuer Alpen ab dem Allerød bis ins Frühholozän folgende Höhenstufung bestanden haben:

Höhenlagen bis ca. $1200 \mathrm{~m}$ ü. NN sind von Kiefernwäldern mit untergeordneter Betula-Partizipation eingenommen.

Lagen bis zur bei ca. $1600 \mathrm{~m}$ ü. NN lokalisierten Waldgrenze sind zusehends arvenreicher.

Darüber sind Krummholzbestände mit Pinus mugo zu vermuten, welche mit zunehmender Höhenlage von Rasengesellschaften und schließlich von Gesteinsschutt- und Felsspaltengesellschaften abgelöst werden.

Für den Kälterückschlag der Jüngeren Dryas ist die schon erwähnte Wald- und Baumgrenzdepression von jeweils etwa $200 \mathrm{~m}$ zu konstatieren.

\subsection{Datierungen}

Für ${ }^{14} \mathrm{C}$-Datierungen am Bohrkern des Unteren Inselsees wurden ausschließlich terrestrische Makrofossilien verwendet. Die konventionellen Radiokarbondaten lassen sich gut mit einigen ${ }^{14} \mathrm{C}$-Datierungen des Schleinsees (MIELKE \& MüLLER 1981: Fig. 6.9) parallelisieren. Vergleicht man die 
kalibrierten Radiokarbondaten (Tab. 4) mit den aktuell existierenden Warvenchronologien vom Meerfelder Maar (LITT \& SteBich 1999, LitT et al. 2001) und vom Steißlinger See (Eusterhues et al. 2002) in Tabelle 6, passen die jüngeren ${ }^{14} \mathrm{C}$-Alter gut in die Zeitschemen. So ergibt sich z.B. für die Grenze Allerød - Jüngere Dryas eine Alterszeitspanne von 12376-12729 cal. BP. Allerdings fällt der ermittelte Zeitraum für die einsetzende Gerzenseeschwankung mit 13431-13763 cal. BP schon deutlich zu alt aus, selbst wenn man allein die Gliederung von Eusterhues et al. (2002) betrachtet. Die bøllingzeitliche Datierung ist bei eben genanntem Zeitschema wiederum schlüssig. Der Grund für fehlerhafte ${ }^{14} \mathrm{C}$-Datierungen in der Jungmoränenlandschaft und den nördlichen Kalkalpen ist meist in Hartwassereffekten zu suchen. RöscH (1990) kommt aufgrund von Vergleichen mit Datierungsergebnissen aus der Schweiz und der Tiefenlage des Laacher See-Tuffs zu dem Schluss, dass die spätglazialen ${ }^{14} \mathrm{C}$-Daten im Bodenseeraum infolge von Hartwassereffekten um 400 bis 600 Jahre zu alt ausfallen. Datierungsfehler aufgrund von umgelagertem Material sind jedoch nicht gänzlich auszuschließen.

\subsection{Bemerkungen zum Meiendorf-Interstadial}

Das Meiendorf wurde erstmals von MENKE (1968) in Schleswig-Holstein beschrieben und ist dort durch einen Anstieg von Betula nana, Hippophaë und Artemisia charakterisiert. Jüngere Arbeiten aus Nord- und Westdeutschland wie z.B. Merkt \& Müller (1999), Schirmer (1999) sowie Stebich (1999) bestätigen diese sinnvolle Abgrenzung, da die letzte Eiszeit ja nicht mit einem erneuten Kälterückschlag (= Ältere Dryas) enden kann. Der Vergleich der Warvenchronologien vom Meerfelder Maar (LITT \& STEBICH 1999, LitT et al. 2001) und vom Steißlinger See (Eusterhues et al. 2002) in Tabelle 6 lehrt jedoch, dass das Meiendorf in Nord- und Westdeutschland dem frühen Bølling bzw. der von BERTSCH (1961: 257) ausgeschiedenen Funiperus-Hippophä̈-Strauchphase in Süddeutschland entspricht. Die süddeutsche Gliederung stimmt mit derjenigen der Schweiz (z.B. AmmanN et al. 1994: 121 und AmmANN et al. 2013: Fig. 7) überein. Im österreichischen Alpenvorland wurde jüngst das Meiendorf das südliche Mitteleuropa betreffend erstmalig abgegrenzt (LAuterbach et al. 2011: Fig. 6). Den eben genannten Publikationen aus Süddeutschland (BERTSCH 1961) und der Schweiz (Ammann et al. 1994, Ammann et al. 2013) zufolge, welche die gültige Chronologie darstellen sollten, ist das Meiendorf größtenteils als Synonym zum ersten Teil des Bøllings zu sehen. Wie bei De KLERK (2004) treffend dargestellt, existieren insbesondere aufgrund von Korrelationsfehlern schon einige verwirrende Unterteilungen der spätglazialen Vegetationsabschnitte im nördlichen Mitteleuropa, welche nicht auch noch auf das südliche Mitteleuropa ausgedehnt werden sollten.

\section{Danksagung}

Wir möchten Herrn Prof. Dr. Hermann Jerz herzlich für dessen tatkräftige Hilfe bei der Wahl des optimalen Bohrpunktes im Gebiet des Unteren Inselsees danken. Zudem gebührt unser Dank den wertvollen Hinweisen der beiden Reviewer.

\section{Literatur}

Ammann, B. \& Lotter, A. (1989): Late-Glacial radiocarbon- and palynostratigraphy on the Swiss Plateau. - Boreas, 18: 109-126.

Ammann, B., Lotter, A., Eicher, U., Gaillard, M.-J., Wohlfahrt, B., Haeberli, W., Lister, G., Maisch, M., Niessen, F. \& Schlüchter, Ch. (1994): The Würmian Late-glacial in lowland Switzerland. - Journal of Quaternary Science, 9 (2): 119-125.

Ammann, B., van Leeuwen, J., van der KnaAp, W., Lischke, H., Heiri, O. \& TINNER, W. (2013): Vegetation responses to rapid warming and to minor climatic fluctuations during the Late-Glacial Interstadial (GI-1) at Gerzensee (Switzerland). - Palaeogeography, Palaeoclimatology, Palaeoecology, 391 (Part B): 40-59.

BADER, K. \& JeRZ, H. (1978): Die glaziale Übertiefung im Iller- und Alpseetal (Oberes Allgäu). - Geologisches Jahrbuch, A46: 25-45.

BeHRE, K.-E. (1978): Die Klimaschwankungen im europäischen Präboreal. Petermanns Geographische Mitteilungen, 122 (2): 97-102.

BERTSCH, A. (1961): Untersuchungen zur spätglazialen Vegetationsgeschichte Südwestdeutschlands. - Flora, 151: 243-280.

Beug, H.-J. (1961): Leitfaden der Pollenbestimmung für Mitteleuropa und angrenzende Gebiete. - 63 S.; Stuttgart (Fischer).

BEUG, H.-J. (1976): Die spät- und frühpostglaziale Vegetationsgeschichte im Gebiet des ehemaligen Rosenheimer Sees (Oberbayern). - Botanische Jahrbücher für Systematik, 95: 373-400.

Beug, H.-J. (2004): Leitfaden der Pollenbestimmung für Mitteleuropa und angrenzende Gebiete. - 542 S.; München (Pfeil).

Bludau, W. (1985): Zur Paläoökologie des Ammergebirges im Spät- und Postglazial. - 363 S.; Rheinfelden (Schäuble).

Bortenschlager, S. (1984): Beiträge zur Vegetationsgeschichte Tirols I. Inneres Öztal und unteres Inntal. - Berichte des NaturwissenschaftlichMedizinischen Vereins in Innsbruck, 71: 19-56.

Bos, J., van Geel, B., van der Pflicht, J. \& Bohncke, S. (2007): Preboreal climate oscillations in Europe: Wiggle-match dating and synthesis of Dutch high-resolution multi-proxy records. - Quaternary Science Reviews, 26: 1927-1950.

Burga, C. $\mho$ Perret, R. (1998): Vegetation und Klima der Schweiz seit dem jüngeren Eiszeitalter. -805 S.; Thun (Ott).

DE KLERK, P. (2004): Confusing concepts in Lateglacial stratigraphy and geochronology: origin, consequences, conclusions (with special emphasis on the type locality Bøllingsø). - Review of Palaeobotany and Palynology, 129: 265-298.

DiefFenBACH-Fries, H. (1981): Zur spät- und postglazialen Vegetationsentwicklung bei Oberstdorf (Oberallgäu) und im Kleinwalsertal (Vorarlberg). Pollen- und makrofossilanalytische Untersuchungen an drei Mooren der montanen Stufe. - 154 S.; Dissertation am Fachbereich Biologie der Technischen Hochschule Darmstadt.

Eicher, U. \& Siegenthaler, U. (1976): Palynological and oxygen isotope investigations on Late-Glacial sediment cores from Swiss lakes. - Boreas, 5: 109-117.

Eusterhues, K., Lechterbeck, J., Schneider, J. \& Wolf-Brozio, U. (2002) Late- and Post-Glacial evolution of Lake Steisslingen (I). Sedimentary history, palynological record and inorganic geochemical indicators. Palaeogeography, Palaeoclimatology, Palaeoecology, 187: 341-371.

FÆgri, K. \& Iversen, J. (1989²): Textbook of Pollen Analysis. - 328 S.; Chichester (Wiley).

GöтtLich, K. (1955): Ein Pollendiagramm ungestörter späteiszeitlicher Verlandungsschichten im Federseebecken. - Beiträge zur naturkundlichen Forschung in Südwestdeutschland, 14: 88-92.

JERZ, H. (1974): Geologische Karte von Bayern 1:25000. Erläuterungen zum Blatt Nr. 8327, Buchenberg. - 181 S.; München (Bayerisches Geologisches Landesamt).

IRMLER, R. \& Scholz, H. (2005): Bestätigt der Fund einer „Schieferkohle“ die spätglaziale Vergletscherung der Immenstädter Nagelfluhberge? - Eiszeitalter und Gegenwart, 55: 88-101.

Ivy-Ochs, S., Kerschner, H., Reuther, A., Preusser, F., Heine, K., Maisch, M., KubIK, P. \& Schlüchter, C. (2008): Chronology of the last glacial cycle in the European Alps. - Journal of Quaternary Science, 23: 559573.

Kleinmann, A. (1995): Seespiegelschwankungen am Ammersee. Ein Beitrag zur spät- und postglazialen Klimageschichte Bayerns. - Geologica Bavarica, 99: 253-367.

KüsteR, H. (1988): Vom Werden einer Kulturlandschaft. Vegetationsgeschichtliche Studien am Auerberg (Südbayern). - 214 S.; Weinheim (Acta humaniora).

LANG, G. (1952): Zur späteiszeitlichen Vegetations- und Florengeschichte Südwestdeutschlands. - Flora, 139: 243-294. 
LANG, G. (1962): Vegetationsgeschichtliche Untersuchungen der Magdalenienstation an der Schussenquelle. - Veröffentlichungen des Geobotanischen Instituts der Eidg. Tech. Hochschule Zürich, 37: 129-154.

LAUTERBACH, J. und mind. 9 weitere (2011): Environmental responses to Lateglacial climatic fluctuations recorded in the sediments of pre-Alpine Lake Mondsee (northeastern Alps). - Journal of Quaternary Science, 26 (3): 253-267.

Lechterbeck, J. (2001): „Human Impact“ oder Climatic Change“? Zur Vegetationsgeschichte des Spätglazials und Holozäns in hochauflösenden Pollenanalysen laminierter Sedimente des Steißlinger Sees (Südwestdeutschland). - Tübinger Mikropaläontologische Mitteilungen, 25: $1-101$.

LitT, T. \& Stebich, M. (1999): Bio- and chronostratigraphy in the Eifel region, Germany. - Quaternary International, 61: 5-16.

Litt, T., Brauer, A., Goslar, T., Merkt, J., Balaga, K., Müller, H., RalsKA-JAsiewiczowa, M., SteBich, M. \& NegendanK, J. (2001): Correlation and synchronisation of Lateglacial continental sequences in northern central Europe based on annually laminated lacustrine sediment. - Quaternary Science Reviews, 20: 1233-1249.

Lotter, A., Eicher, U., Siegenthaler, U., \& Birks, H.J.B. (1992): Late Glacial climatic oscillations as recorded in Swiss lake sediments. - Journal of Quaternary Science, 7 (3): 187-204.

MerkT, J. \& MülLer, H. (1999): Varve chronology and palynology of the Lateglacial in Northwest Germany from lacustrine sediments of Hämelsee in Lower Saxony. - Quaternary International, 61: 41-59.

MielKe, K. \& Müller, H. (1981): Palynologie. - In: Bender, F. (ed.): Angewandte Geowissenschaften 1: 393-407; Stuttgart (Enke).

Moore, P., WebB, J. \& Collinson, M. (19912): Pollen Analysis. - 216 S.; London (Blackwell)

MüLleR, H. (1962): Pollenanalytische Untersuchung eines Quartärprofils durch die spät- und nacheiszeitlichen Ablagerungen des Schleinsees (Südwestdeutschland). - Geologisches Jahrbuch, 79: 493-526.

ОвiDowicz, А. \& SсновеR, Н. (1985): Moorkundliche und vegetationsgeschichtliche Untersuchungen des Sennalpenmoores im Trauchgauer Flysch (Ammergebirge). - Berichte der Bayerischen Botanischen Gesellschaft, 56: 147-165.

Paul, H. \& Ruoff, S. (1932): Pollenstatistische und stratigraphische Mooruntersuchungen im südlichen Bayern. II. Teil. Moore in den Gebieten der Isar-, Allgäu- und Rheinvorlandgletscher. - Berichte der Bayerischen Botanischen Gesellschaft, 20: 1-264.

RAusch, K. (1975): Untersuchungen zur spät- und nacheiszeitlichen Vegetationsgeschichte im Gebiet des ehemaligen Inn-Chiemseegletschers. - Flora, 164: 235-282.

Reille, M. (1998): Pollen et Spores d'Europe et d'Afrique du Nord. Supplement 2. - 521 S.; Marseille (Laboratoire de Botanique historique et Palynologie).

ReIMER, P.J. und 29 weitere (2013): IntCal13 and Marine13 radiocarbon age calibration curves, 0-50,000 years cal BP. - Radiocarbon, 55 (4): 18691187.
ReITNER, J. (2007): Glacial dynamics at the beginning of Termination I in the Eastern Alps and their stratigraphic implications. - Quaternary International, 164-165: 64-84.

Rösch, M. (1985): Ein Pollenprofil aus dem Feuenried bei Überlingen am Ried: Stratigraphische und landschaftsgeschichtliche Bedeutung für das Holozän im Bodenseegebiet. - Materialhefte zur Vor- und Frühgeschichte in Baden-Württemberg, 7 (Berichte zu Ufer- und Moorsiedlungen Südwestdeutschlands 2): 43-79.

Rösch, M. (1990): Vegetationsgeschichtliche Untersuchungen im Durchenbergried. - Siedlungsarchäologie im Alpenvorland II. - Forschungen und Berichte zur Vor- und Frühgeschichte in Baden-Württemberg, 37: $9-64$.

SchmeIdL, H. (1971): Ein Beitrag zur spätglazialen Vegetations- und Waldentwicklung im westlichen Salzachgletschergebiet. - Eiszeitalter und Gegenwart, 22: 110-126.

Schirmer, U. (1999): Pollenstratigraphische Gliederung des Spätglazials im Rheinland. - Eiszeitalter und Gegenwart, 49: 132-143.

Scholz, H. $\left(1995^{2}\right)$ : Bau und Werden der Allgäuer Landschaft. - 305 S Stuttgart (Schweizerbart).

SteBICH, M. (1999): Palynologische Untersuchungen zur Vegetationsgeschichte des Weichsel-Spätglazial und Frühholozän an jährlich geschichteten Sedimenten des Meerfelder Maares (Eifel). - Dissertationes Botanicae, 320: 1-127.

Stebich, M., Mingram, J., Han, J. \& LiU, J. (2009): Late Pleistocene spread of (cool-)temperate forests in Northeast China and climate changes synchronous with the North Atlantic region. - Global and Planetary Change, 65: 56-70

STOCKMARR, J. (1971): Tablets with spores used in absolute pollen analysis. - Pollen et Spores, 13: 615-621.

Stuiver, M. \& Reimer, P.J. (1993): Extended ${ }^{14} \mathrm{C}$ database and revised CALIB radiocarbon calibration program. - Radiocarbon, 35: 215-230.

van Mourik, J., Slotboom, R., van der Pflicht, J., Streurman, H., KuiJPER, W., Hoek, W. \& DE GRAAF, L. (2013): Geochronology of Betula extensions in pollen diagrams of Alpine Late-glacial lake deposits: a case study of the Late-glacial deposits of the Gasserplatz soil archives (Vorarlberg, Austria). - Quaternary International, 306: 3-13.

VöLK, H. (2001): Geomorphologie des Kleinwalsertales und seiner Gebirgsumrahmung. Landschaftsformen zur Eiszeit und Nacheiszeit unter Einbeziehung der geologischen Verhältnisse. - Vorarlberger Naturschau, 10: 7-95.

VoIGT, R. (1996): Paläolimnologische und vegetationsgeschichtliche Untersuchungen an Sedimenten aus Fuschlsee und Chiemsee (Salzburg und Bayern). - Dissertationes Botanicae, 270: 1-303.

WAHLMÜLLER, N. (1985): Beiträge zur Vegetationsgeschichte Tirols V: Nordtiroler Kalkalpen. - Berichte des Naturwissenschaftlich-Medizinischen Vereins in Innsbruck, 72: 101-144.

WICK, L. (2000): Vegetational response to climatic changes recorded in Swiss Late Glacial lake sediments. - Palaeogeography, Palaeoclimatology, Palaeoecology, 159: 231-250. 